\title{
Photoconducting Devices with Response in the Visible-Near-Infrared Region Based on Neutral Ni Complexes of Aryl-1,2-dithiolene Ligands
}

Anna Pintus, ${ }^{* a}$ Lucia Ambrosio, ${ }^{a}$ M. Carla Aragoni, ${ }^{a}$ Maddalena Binda,,${ }^{\text {b }}$ Simon J. Coles, ${ }^{\mathrm{c}}$ Michael B. Hursthouse, ${ }^{\mathrm{c}}$ Francesco Isaia, ${ }^{\mathrm{a}}$ Vito Lippolis, ${ }^{\mathrm{a}}$ Giammarco Meloni, ${ }^{\mathrm{a}}$ Dario Natali,,${ }^{\mathrm{b} d} \mathrm{James}$ B. Orton, ${ }^{\mathrm{c}}$ Enrico Podda,${ }^{\mathrm{a}}$ Marco Sampietro, ${ }^{\mathrm{b}, \mathrm{d}}$ and Massimiliano Arca*a

${ }^{a}$ Università degli Studi di Cagliari, Dipartimento di Scienze Chimiche e Geologiche, S.S. 554 bivio per Sestu,09042 Monserrato (Cagliari),Italy.E-mail: apintus@unica.it,marca@unica.it

${ }^{b}$ Center for Nano Science and Technology@Polimi, Istituto Italiano di Tecnologia, Via Pascoli 70/3, 20133 Milano, Italy

${ }^{c}$ UK National Crystallography Service, School of Chemistry, Faculty of Engineering and Physical Sciences, University of Southampton, SO17 1BJ, United Kingdom

${ }^{d}$ Politecnico di Milano, Dipartimento di Elettronica, Informazione e Bioingegneria, Via Ponzio 34/5, 20133 Milano, Italy

¥ Current affiliation: Energy Everywhere Italy, Via Pascoli 70/3, 20133 Milano, Italy 
Table S1. Crystal data for 4-phenyl-[1,3]dithiol-2-one, ${ }^{\mathrm{a}} \mathbf{1},{ }^{\mathrm{b}} \mathbf{2},{ }^{\mathrm{c}}\left(\mathrm{TBA}^{+}\right)\left(\mathbf{1}^{-}\right),{ }^{\mathrm{b}}$ and $\left(\mathrm{TBA}^{+}\right)\left(\mathbf{2}^{-}\right) .{ }^{\mathrm{c}}$

\begin{tabular}{|c|c|c|c|c|c|}
\hline & $\begin{array}{c}\text { 4-phenyl- } \\
{[1,3] \text { dithiol-2-one }}\end{array}$ & 1 & 2 & $\left(\mathrm{TBA}^{+}\right)\left(\mathbf{1}^{-}\right)$ & $\left(\mathrm{TBA}^{+}\right)\left(\mathbf{2}^{-}\right)$ \\
\hline Empirical formula & $\mathrm{C}_{9} \mathrm{H}_{6} \mathrm{OS}_{2}$ & $\mathrm{C}_{16} \mathrm{H}_{12} \mathrm{~S}_{4} \mathrm{Ni}$ & $\mathrm{C}_{24} \mathrm{H}_{16} \mathrm{~S}_{4} \mathrm{Ni}$ & $\mathrm{C}_{32} \mathrm{H}_{48} \mathrm{NS}_{4} \mathrm{Ni}$ & $\mathrm{C}_{40} \mathrm{H}_{52} \mathrm{NS}_{4} \mathrm{Ni}$ \\
\hline$M_{\mathrm{r}}$ & 194.26 & 391.21 & 491.32 & 633.66 & 733.78 \\
\hline Crystal size (mm) & $0.36 \times 0.12 \times 0.02$ & $0.15 \times 0.01 \times 0.01$ & $0.15 \times 0.08 \times 0.01$ & $0.24 \times 0.14 \times 0.02$ & $0.15 \times 0.02 \times 0.02$ \\
\hline Crystal system & Orthorhombic & Monoclinic & Monoclinic & Monoclinic & Orthorhombic \\
\hline Space group & Pbca & $P 2_{1} / \mathrm{c}$ & $P 2_{1} / \mathrm{c}$ & $C 2 / c$ & $P 2{ }_{1}{ }_{1} 2_{1}$ \\
\hline$a(\AA)$ & $10.3779(6)$ & $9.2787(10)$ & $20.3162(14)$ & $19.9008(6)$ & $12.113(5)$ \\
\hline$b(\AA)$ & $7.8316(3)$ & $9.4236(10)$ & $6.0502(2)$ & $8.6982(2)$ & $16.307(6)$ \\
\hline$c(\AA)$ & $20.3844(12)$ & $17.749(2)$ & $7.9863(3)$ & $19.3097(5)$ & $19.257(8)$ \\
\hline$V\left(\AA^{3}\right)$ & $1656.75(15)$ & $1551.7(3)$ & $975.43(8)$ & $3183.18(15)$ & $3804(3)$ \\
\hline$Z$ & 8 & 4 & 2 & 4 & 4 \\
\hline Calculated density $\left(\mathrm{mg} \mathrm{m}^{-3}\right)$ & 1.558 & 1.675 & 1.673 & 1.322 & 1.281 \\
\hline Total number of data & 10683 & 9241 & 6749 & 32826 & 21880 \\
\hline Unique reflections & 1900 & 2724 & 2222 & 3652 & 7715 \\
\hline$R_{\text {int }}$ & 0.0594 & 0.0995 & 0.0399 & 0.0348 & 0.0679 \\
\hline Parameters & 109 & 194 & 133 & 176 & 420 \\
\hline Final $R$ indeces $(I>2 \sigma(I))$ & 0.0400 & 0.0709 & 0.0334 & 0.0314 & 0.0529 \\
\hline$w R 2$ (all data) & 0.0966 & 0.2021 & 0.0836 & 0.0756 & 0.1317 \\
\hline
\end{tabular}

${ }^{\mathrm{a}} \mathrm{T}=120(2) \mathrm{K}$. MoK $\alpha$ radiation $(\lambda=0.71075 \AA) ;{ }^{\mathrm{b}} \mathrm{T}=100(2) \mathrm{K}$. MoK $\alpha$ radiation $(\lambda=0.71075 \AA) ;{ }^{\mathrm{c}} \mathrm{T}=120(2) \mathrm{K}$. MoKa radiation $(\lambda=0.69050 \AA)$. 
Table S2. Electrochemical data $\left(\mathrm{V} v s \mathrm{Fc}^{+} / \mathrm{Fc}\right)$ for $\left(\mathrm{TBA}^{+}\right)\left(\mathbf{1}^{-}\right)$and $\left(\mathrm{TBA}^{+}\right)\left(\mathbf{2}^{-}\right)\left(25^{\circ} \mathrm{C}\right.$; scan rate $0.100 \mathrm{~V} \mathrm{~s}^{-1}$; supporting electrolyte $\left.\left(\mathrm{TBA}^{+}\right)\left(\mathrm{PF}_{6}^{-}\right) 0.1 \mathrm{M}\right)$.

\begin{tabular}{cccc}
\hline & & $\left(\mathrm{TBA}^{+}\right)\left(\mathbf{1}^{-}\right)$ & $\left(\mathrm{TBA}^{+}\right)\left(\mathbf{2}^{-}\right)$ \\
\hline & $E_{1 / 2}$ & -1.24 & $-1.21 \mathrm{~V}$ \\
$(-2 /-1)$ & $\left|E_{\mathrm{pc}}-E_{\mathrm{pa}}\right|$ & $4.0 \cdot 10^{-2}$ & $7.0 \cdot 10^{-2}$ \\
& $i_{\mathrm{pc}} / i_{\mathrm{pa}}$ & 0.95 & 0.70 \\
& $E_{1 / 2}$ & -0.41 & -0.41 \\
$(-1 / 0)$ & $\left|E_{\mathrm{pc}}-E_{\mathrm{pa}}\right|$ & $5.0 \cdot 10^{-2}$ & $8.0 \cdot 10^{-2}$ \\
& $i_{\mathrm{pc}} / i_{\mathrm{pa}}$ & 1.06 & 1.05 \\
\hline
\end{tabular}


Table S3. Optimised geometry calculated for $\mathbf{1}$ (cis conformation) at DFT level in the gas phase (total charge $=0$, spin multiplicity $=1$ ) in orthogonal Cartesian coordinate format.

\begin{tabular}{|c|c|c|c|c|}
\hline Atom number & $Z$ & $\mathrm{X}$ & $\mathrm{Y}$ & Z \\
\hline 1 & 6 & 0.624361 & -4.316737 & -1.835011 \\
\hline 2 & 6 & 0.032217 & -4.244701 & -0.563073 \\
\hline 3 & 6 & -0.551628 & -5.407610 & -0.031314 \\
\hline 4 & 6 & -0.536641 & -6.600972 & -0.745298 \\
\hline 5 & 6 & 0.059604 & -6.659117 & -2.004404 \\
\hline 6 & 6 & 0.638108 & -5.511587 & -2.545847 \\
\hline 7 & 6 & 0.019055 & -2.979621 & 0.193919 \\
\hline 8 & 6 & 0.024928 & -2.942240 & 1.584047 \\
\hline 9 & 16 & 0.011046 & -1.494643 & 2.446373 \\
\hline 10 & 28 & 0.000000 & 0.000000 & 0.899475 \\
\hline 11 & 16 & -0.011046 & 1.494643 & 2.446373 \\
\hline 12 & 6 & -0.024928 & 2.942240 & 1.584047 \\
\hline 13 & 6 & -0.019055 & 2.979621 & 0.193919 \\
\hline 14 & 6 & -0.032217 & 4.244701 & -0.563073 \\
\hline 15 & 6 & 0.551628 & 5.407610 & -0.031314 \\
\hline 16 & 6 & 0.536641 & 6.600972 & -0.745299 \\
\hline 17 & 6 & -0.059604 & 6.659117 & -2.004404 \\
\hline 18 & 6 & -0.638108 & 5.511587 & -2.545848 \\
\hline 19 & 6 & -0.624361 & 4.316737 & -1.835012 \\
\hline 20 & 16 & 0.001861 & -1.492040 & -0.652136 \\
\hline 21 & 16 & -0.001861 & 1.492040 & -0.652136 \\
\hline 22 & 1 & -0.072182 & 3.865491 & 2.167390 \\
\hline 23 & 1 & 1.049038 & 5.370766 & 0.938831 \\
\hline 24 & 1 & 1.002909 & 7.490498 & -0.318389 \\
\hline 25 & 1 & -0.071278 & 7.596299 & -2.563300 \\
\hline 26 & 1 & -1.110017 & 5.548121 & -3.529111 \\
\hline 27 & 1 & -1.088492 & 3.424238 & -2.257470 \\
\hline 28 & 1 & -1.049038 & -5.370766 & 0.938831 \\
\hline 29 & 1 & -1.002909 & -7.490498 & -0.318388 \\
\hline 30 & 1 & 0.071278 & -7.596299 & -2.563300 \\
\hline 31 & 1 & 1.110017 & -5.548121 & -3.529111 \\
\hline 32 & 1 & 1.088492 & -3.424239 & -2.257470 \\
\hline 33 & 1 & 0.072182 & -3.865491 & 2.167390 \\
\hline
\end{tabular}


Table S4. Optimised geometry calculated for 1 (trans conformation) at DFT level in the gas phase (total charge $=0$, spin multiplicity $=1$ ) in orthogonal Cartesian coordinate format.

\begin{tabular}{|c|c|c|c|c|}
\hline Atom number & $Z$ & $\mathrm{X}$ & $\mathrm{Y}$ & Z \\
\hline 1 & 28 & 0.000000 & 0.000000 & -0.008253 \\
\hline 2 & 16 & -0.859815 & -1.970608 & -0.007528 \\
\hline 3 & 16 & -1.941152 & 0.932913 & 0.000960 \\
\hline 4 & 16 & 1.941152 & -0.932913 & 0.000960 \\
\hline 5 & 16 & 0.859815 & 1.970608 & -0.007528 \\
\hline 6 & 6 & 3.039720 & 0.379504 & 0.001841 \\
\hline 7 & 6 & 2.517570 & 1.668397 & -0.004362 \\
\hline 8 & 6 & -2.517570 & -1.668397 & -0.004362 \\
\hline 9 & 6 & -3.039720 & -0.379504 & 0.001841 \\
\hline 10 & 1 & 3.178240 & 2.538490 & -0.040982 \\
\hline 11 & 1 & -3.178240 & -2.538490 & -0.040982 \\
\hline 12 & 6 & 4.489673 & 0.113062 & 0.003046 \\
\hline 13 & 6 & -4.489673 & -0.113062 & 0.003046 \\
\hline 14 & 6 & 5.387446 & 1.018448 & 0.595023 \\
\hline 15 & 6 & 6.755253 & 0.767089 & 0.593483 \\
\hline 16 & 6 & 7.256014 & -0.392519 & 0.002900 \\
\hline 17 & 6 & 6.376071 & -1.301611 & -0.583508 \\
\hline 18 & 6 & 5.007990 & -1.053693 & -0.583231 \\
\hline 19 & 1 & 5.008827 & 1.914723 & 1.088239 \\
\hline 20 & 1 & 7.434638 & 1.478518 & 1.065941 \\
\hline 21 & 1 & 8.329556 & -0.588269 & 0.001877 \\
\hline 22 & 1 & 6.758821 & -2.210377 & -1.050929 \\
\hline 23 & 1 & 4.324325 & -1.762244 & -1.053308 \\
\hline 24 & 6 & -5.387446 & -1.018448 & 0.595023 \\
\hline 25 & 6 & -6.755253 & -0.767089 & 0.593483 \\
\hline 26 & 6 & -7.256014 & 0.392519 & 0.002900 \\
\hline 27 & 6 & -6.376071 & 1.301611 & -0.583508 \\
\hline 28 & 6 & -5.007990 & 1.053693 & -0.583231 \\
\hline 29 & 1 & -5.008827 & -1.914723 & 1.088239 \\
\hline 30 & 1 & -7.434638 & -1.478518 & 1.065941 \\
\hline 31 & 1 & -8.329556 & 0.588269 & 0.001877 \\
\hline 32 & 1 & -6.758821 & 2.210377 & -1.050929 \\
\hline 33 & 1 & -4.324325 & 1.762244 & -1.053308 \\
\hline
\end{tabular}


Table S5. Optimised geometry calculated for $\mathbf{1}^{-}$( cis conformation) at DFT level in the gas phase (total charge $=-1$, spin multiplicity $=2$ ) in orthogonal Cartesian coordinate format.

\begin{tabular}{|c|c|c|c|c|}
\hline Atom number & $Z$ & $\mathrm{X}$ & $\mathrm{Y}$ & Z \\
\hline 1 & 6 & -4.340769 & -0.526610 & 1.862380 \\
\hline 2 & 6 & -4.287300 & -0.010413 & 0.554624 \\
\hline 3 & 6 & -5.482369 & 0.497800 & 0.010652 \\
\hline 4 & 6 & -6.673307 & 0.470750 & 0.729322 \\
\hline 5 & 6 & -6.708867 & -0.053470 & 2.021834 \\
\hline 6 & 6 & -5.531268 & -0.547760 & 2.582390 \\
\hline 7 & 6 & -3.025864 & -0.001545 & -0.209856 \\
\hline 8 & 6 & -2.990018 & -0.009294 & -1.579632 \\
\hline 9 & 16 & -1.525111 & -0.008729 & -2.462917 \\
\hline 10 & 28 & 0.000000 & 0.000000 & -0.905389 \\
\hline 11 & 16 & 1.525111 & 0.008729 & -2.462917 \\
\hline 12 & 6 & 2.990018 & 0.009294 & -1.579632 \\
\hline 13 & 6 & 3.025864 & 0.001545 & -0.209856 \\
\hline 14 & 6 & 4.287300 & 0.010413 & 0.554624 \\
\hline 15 & 6 & 5.482369 & -0.497800 & 0.010652 \\
\hline 16 & 6 & 6.673307 & -0.470750 & 0.729322 \\
\hline 17 & 6 & 6.708867 & 0.053470 & 2.021834 \\
\hline 18 & 6 & 5.531268 & 0.547760 & 2.582390 \\
\hline 19 & 6 & 4.340769 & 0.526610 & 1.862380 \\
\hline 20 & 16 & -1.516689 & 0.002347 & 0.657063 \\
\hline 21 & 16 & 1.516689 & -0.002347 & 0.657063 \\
\hline 22 & 1 & 3.912250 & 0.052486 & -2.166451 \\
\hline 23 & 1 & 5.468026 & -0.942975 & -0.985215 \\
\hline 24 & 1 & 7.581978 & -0.877243 & 0.278875 \\
\hline 25 & 1 & 7.642510 & 0.069156 & 2.587992 \\
\hline 26 & 1 & 5.538687 & 0.959598 & 3.594284 \\
\hline 27 & 1 & 3.422850 & 0.919193 & 2.302716 \\
\hline 28 & 1 & -5.468026 & 0.942975 & -0.985215 \\
\hline 29 & 1 & -7.581978 & 0.877243 & 0.278875 \\
\hline 30 & 1 & -7.642510 & -0.069156 & 2.587992 \\
\hline 31 & 1 & -5.538687 & -0.959598 & 3.594284 \\
\hline 32 & 1 & -3.422850 & -0.919193 & 2.302716 \\
\hline 33 & 1 & -3.912250 & -0.052486 & -2.166451 \\
\hline
\end{tabular}


Table S6. Optimised geometry calculated for $\mathbf{1}^{-}$(trans conformation) at DFT level in the gas phase (total charge $=-$ 1 , spin multiplicity $=2$ ) in orthogonal Cartesian coordinate format.

\begin{tabular}{|c|c|c|c|c|}
\hline Atom number & $Z$ & $\mathrm{X}$ & $\mathrm{Y}$ & $\mathrm{Z}$ \\
\hline 1 & 28 & 0.000000 & 0.000000 & -0.013801 \\
\hline 2 & 16 & -0.889174 & 1.991303 & -0.009595 \\
\hline 3 & 16 & -1.964249 & -0.937648 & -0.016831 \\
\hline 4 & 16 & 1.964249 & 0.937648 & -0.016831 \\
\hline 5 & 16 & 0.889174 & -1.991303 & -0.009595 \\
\hline 6 & 6 & 3.079379 & -0.398794 & -0.013751 \\
\hline 7 & 6 & 2.569288 & -1.670592 & -0.007693 \\
\hline 8 & 6 & -2.569288 & 1.670592 & -0.007693 \\
\hline 9 & 6 & -3.079379 & 0.398794 & -0.013751 \\
\hline 10 & 1 & 3.230050 & -2.541412 & 0.035036 \\
\hline 11 & 1 & -3.230050 & 2.541412 & 0.035036 \\
\hline 12 & 6 & 4.527688 & -0.120155 & -0.003191 \\
\hline 13 & 6 & -4.527688 & 0.120155 & -0.003191 \\
\hline 14 & 6 & 5.460174 & -1.045356 & -0.510084 \\
\hline 15 & 6 & 6.826547 & -0.785118 & -0.481025 \\
\hline 16 & 6 & 7.308183 & 0.414501 & 0.043983 \\
\hline 17 & 6 & 6.397895 & 1.349218 & 0.536894 \\
\hline 18 & 6 & 5.031456 & 1.087604 & 0.513662 \\
\hline 19 & 1 & 5.101499 & -1.974277 & -0.955715 \\
\hline 20 & 1 & 7.522730 & -1.523201 & -0.886456 \\
\hline 21 & 1 & 8.380339 & 0.621009 & 0.061294 \\
\hline 22 & 1 & 6.755777 & 2.295502 & 0.949322 \\
\hline 23 & 1 & 4.323071 & 1.819392 & 0.905156 \\
\hline 24 & 6 & -5.460174 & 1.045356 & -0.510084 \\
\hline 25 & 6 & -6.826547 & 0.785118 & -0.481025 \\
\hline 26 & 6 & -7.308183 & -0.414501 & 0.043983 \\
\hline 27 & 6 & -6.397895 & -1.349218 & 0.536894 \\
\hline 28 & 6 & -5.031456 & -1.087604 & 0.513662 \\
\hline 29 & 1 & -5.101499 & 1.974277 & -0.955715 \\
\hline 30 & 1 & -7.522730 & 1.523201 & -0.886456 \\
\hline 31 & 1 & -8.380339 & -0.621009 & 0.061294 \\
\hline 32 & 1 & -6.755777 & -2.295502 & 0.949322 \\
\hline 33 & 1 & -4.323071 & -1.819392 & 0.905156 \\
\hline
\end{tabular}


Table S7. Optimised geometry calculated for 2 (cis conformation) at DFT level in the gas phase (total charge $=0$, spin multiplicity $=1$ ) in orthogonal Cartesian coordinate format.

\begin{tabular}{|c|c|c|c|c|}
\hline Atom number & $Z$ & $\mathrm{X}$ & $\mathrm{Y}$ & $\mathrm{Z}$ \\
\hline 1 & 6 & -4.269337 & -0.828874 & 1.533410 \\
\hline 2 & 6 & -4.237663 & -0.231013 & 0.240342 \\
\hline 3 & 6 & -5.414892 & 0.283942 & -0.280994 \\
\hline 4 & 6 & -6.634704 & 0.220898 & 0.433226 \\
\hline 5 & 6 & -6.653449 & -0.390873 & 1.724771 \\
\hline 6 & 6 & -5.437926 & -0.907878 & 2.245323 \\
\hline 7 & 6 & -2.976486 & -0.158317 & -0.516682 \\
\hline 8 & 16 & -1.490366 & -0.077726 & 0.329841 \\
\hline 9 & 28 & 0.000000 & 0.000000 & -1.221990 \\
\hline 10 & 16 & 1.490366 & 0.077726 & 0.329841 \\
\hline 11 & 6 & 2.976486 & 0.158317 & -0.516682 \\
\hline 12 & 6 & 2.938453 & 0.158785 & -1.907114 \\
\hline 13 & 16 & 1.493201 & 0.076836 & -2.768764 \\
\hline 14 & 6 & 4.237663 & 0.231013 & 0.240342 \\
\hline 15 & 6 & 4.269337 & 0.828874 & 1.533410 \\
\hline 16 & 6 & 5.437926 & 0.907878 & 2.245323 \\
\hline 17 & 6 & 6.653449 & 0.390873 & 1.724771 \\
\hline 18 & 6 & 6.634704 & -0.220898 & 0.433226 \\
\hline 19 & 6 & 5.414892 & -0.283942 & -0.280994 \\
\hline 20 & 16 & -1.493201 & -0.076836 & -2.768764 \\
\hline 21 & 6 & -2.938453 & -0.158785 & -1.907114 \\
\hline 22 & 1 & -3.858446 & -0.247710 & -2.490673 \\
\hline 23 & 1 & -5.412994 & 0.780470 & -1.252862 \\
\hline 24 & 1 & -5.444971 & -1.380304 & 3.229553 \\
\hline 25 & 1 & -3.347110 & -1.239353 & 1.946589 \\
\hline 26 & 1 & 5.412994 & -0.780470 & -1.252862 \\
\hline 27 & 1 & 5.444971 & 1.380304 & 3.229553 \\
\hline 28 & 1 & 3.347110 & 1.239353 & 1.946589 \\
\hline 29 & 1 & 3.858446 & 0.247710 & -2.490673 \\
\hline 30 & 6 & 7.842429 & -0.751946 & -0.092531 \\
\hline 31 & 6 & 9.013902 & -0.674217 & 0.622722 \\
\hline 32 & 6 & 9.031885 & -0.063502 & 1.898071 \\
\hline 33 & 6 & 7.877151 & 0.456633 & 2.436152 \\
\hline 34 & 1 & 7.826502 & -1.223244 & -1.077364 \\
\hline 35 & 1 & 9.935841 & -1.084724 & 0.207587 \\
\hline 36 & 1 & 9.967967 & -0.007726 & 2.456279 \\
\hline 37 & 1 & 7.889304 & 0.926475 & 3.421647 \\
\hline 38 & 6 & -7.842429 & 0.751946 & -0.092531 \\
\hline 39 & 6 & -9.013902 & 0.674217 & 0.622722 \\
\hline 40 & 6 & -9.031885 & 0.063502 & 1.898071 \\
\hline 41 & 6 & -7.877151 & -0.456633 & 2.436152 \\
\hline 42 & 1 & -7.826502 & 1.223244 & -1.077364 \\
\hline 43 & 1 & -9.935841 & 1.084724 & 0.207587 \\
\hline 44 & 1 & -9.967967 & 0.007726 & 2.456279 \\
\hline 45 & 1 & -7.889304 & -0.926475 & 3.421647 \\
\hline
\end{tabular}


Table S8. Optimised geometry calculated for 2 (trans conformation) at DFT level in the gas phase (total charge $=0$, spin multiplicity $=1$ ) in orthogonal Cartesian coordinate format.

\begin{tabular}{|c|c|c|c|c|}
\hline Atom number & $Z$ & $\mathrm{X}$ & $\mathrm{Y}$ & $\mathrm{Z}$ \\
\hline 1 & 6 & -4.946945 & -1.330592 & 0.701741 \\
\hline 2 & 6 & -4.490403 & -0.092398 & 0.163680 \\
\hline 3 & 6 & -5.425416 & 0.780294 & -0.372843 \\
\hline 4 & 6 & -6.806602 & 0.474112 & -0.388200 \\
\hline 5 & 6 & -7.250078 & -0.767943 & 0.162426 \\
\hline 6 & 6 & -6.279160 & -1.652278 & 0.701881 \\
\hline 7 & 6 & -3.055976 & 0.240725 & 0.173075 \\
\hline 8 & 6 & -2.591253 & 1.552023 & 0.184511 \\
\hline 9 & 16 & -0.949603 & 1.928937 & 0.190059 \\
\hline 10 & 28 & 0.000000 & 0.000000 & 0.187022 \\
\hline 11 & 16 & 0.949603 & -1.928937 & 0.190059 \\
\hline 12 & 6 & 2.591253 & -1.552023 & 0.184511 \\
\hline 13 & 6 & 3.055976 & -0.240725 & 0.173075 \\
\hline 14 & 6 & 4.490403 & 0.092398 & 0.163680 \\
\hline 15 & 6 & 5.425416 & -0.780294 & -0.372843 \\
\hline 16 & 6 & 6.806602 & -0.474112 & -0.388200 \\
\hline 17 & 6 & 7.250078 & 0.767943 & 0.162426 \\
\hline 18 & 6 & 6.279160 & 1.652278 & 0.701881 \\
\hline 19 & 6 & 4.946945 & 1.330592 & 0.701741 \\
\hline 20 & 16 & -1.896952 & -1.020147 & 0.178278 \\
\hline 21 & 16 & 1.896952 & 1.020147 & 0.178278 \\
\hline 22 & 1 & 3.289649 & -2.391878 & 0.223472 \\
\hline 23 & 1 & -3.289649 & 2.391878 & 0.223472 \\
\hline 24 & 1 & 5.103570 & -1.719560 & -0.825604 \\
\hline 25 & 1 & 6.610009 & 2.601446 & 1.127990 \\
\hline 26 & 1 & 4.217723 & 2.019848 & 1.129243 \\
\hline 27 & 1 & -5.103570 & 1.719560 & -0.825604 \\
\hline 28 & 1 & -6.610009 & -2.601446 & 1.127990 \\
\hline 29 & 1 & -4.217723 & -2.019848 & 1.129243 \\
\hline 30 & 6 & 8.634077 & 1.070949 & 0.148454 \\
\hline 31 & 6 & 9.541625 & 0.187045 & -0.388793 \\
\hline 32 & 6 & 9.104187 & -1.040424 & -0.938072 \\
\hline 33 & 6 & 7.767778 & -1.362995 & -0.938893 \\
\hline 34 & 1 & 8.970061 & 2.019883 & 0.571167 \\
\hline 35 & 1 & 10.605106 & 0.431894 & -0.394307 \\
\hline 36 & 1 & 9.833519 & -1.732250 & -1.362681 \\
\hline 37 & 1 & 7.428085 & -2.310176 & -1.362877 \\
\hline 38 & 6 & -7.767778 & 1.362995 & -0.938893 \\
\hline 39 & 6 & -9.104187 & 1.040424 & -0.938072 \\
\hline 40 & 6 & -9.541625 & -0.187045 & -0.388793 \\
\hline 41 & 6 & -8.634077 & -1.070949 & 0.148454 \\
\hline 42 & 1 & -7.428085 & 2.310176 & -1.362877 \\
\hline 43 & 1 & -9.833519 & 1.732250 & -1.362681 \\
\hline 44 & 1 & -10.605106 & -0.431894 & -0.394307 \\
\hline 45 & 1 & -8.970061 & -2.019883 & 0.571167 \\
\hline
\end{tabular}


Table S9. Optimised geometry calculated for $\mathbf{2}^{-}$( cis conformation) at DFT level in the gas phase (total charge $=-1$, spin multiplicity $=2$ ) in orthogonal Cartesian coordinate format.

\begin{tabular}{|c|c|c|c|c|}
\hline Atom number & $Z$ & $\mathrm{X}$ & $\mathrm{Y}$ & $\mathrm{Z}$ \\
\hline 1 & 6 & -4.307691 & -0.782374 & 1.521110 \\
\hline 2 & 6 & -4.279830 & -0.190408 & 0.222647 \\
\hline 3 & 6 & -5.471259 & 0.312609 & -0.282178 \\
\hline 4 & 6 & -6.687997 & 0.227286 & 0.435048 \\
\hline 5 & 6 & -6.697726 & -0.382505 & 1.728965 \\
\hline 6 & 6 & -5.470113 & -0.876499 & 2.242603 \\
\hline 7 & 6 & -3.020702 & -0.128716 & -0.538830 \\
\hline 8 & 16 & -1.514208 & -0.070408 & 0.330899 \\
\hline 9 & 28 & 0.000000 & 0.000000 & -1.231832 \\
\hline 10 & 16 & 1.514208 & 0.070408 & 0.330899 \\
\hline 11 & 6 & 3.020702 & 0.128716 & -0.538830 \\
\hline 12 & 6 & 2.985624 & 0.127912 & -1.909228 \\
\hline 13 & 16 & 1.522603 & 0.067261 & -2.790491 \\
\hline 14 & 6 & 4.279830 & 0.190408 & 0.222647 \\
\hline 15 & 6 & 4.307691 & 0.782374 & 1.521110 \\
\hline 16 & 6 & 5.470113 & 0.876499 & 2.242603 \\
\hline 17 & 6 & 6.697726 & 0.382505 & 1.728965 \\
\hline 18 & 6 & 6.687997 & -0.227286 & 0.435048 \\
\hline 19 & 6 & 5.471259 & -0.312609 & -0.282178 \\
\hline 20 & 16 & -1.522603 & -0.067261 & -2.790491 \\
\hline 21 & 6 & -2.985624 & -0.127912 & -1.909228 \\
\hline 22 & 1 & -3.906958 & -0.206985 & -2.493851 \\
\hline 23 & 1 & -5.477458 & 0.811488 & -1.252921 \\
\hline 24 & 1 & -5.462425 & -1.342813 & 3.230762 \\
\hline 25 & 1 & -3.371846 & -1.170092 & 1.926395 \\
\hline 26 & 1 & 5.477458 & -0.811488 & -1.252921 \\
\hline 27 & 1 & 5.462425 & 1.342813 & 3.230762 \\
\hline 28 & 1 & 3.371846 & 1.170092 & 1.926395 \\
\hline 29 & 1 & 3.906958 & 0.206985 & -2.493851 \\
\hline 30 & 6 & 7.909443 & -0.738635 & -0.081653 \\
\hline 31 & 6 & 9.078957 & -0.645949 & 0.636653 \\
\hline 32 & 6 & 9.086383 & -0.037084 & 1.913252 \\
\hline 33 & 6 & 7.918608 & 0.463598 & 2.443797 \\
\hline 34 & 1 & 7.903561 & -1.208813 & -1.067690 \\
\hline 35 & 1 & 10.007507 & -1.043380 & 0.221419 \\
\hline 36 & 1 & 10.019432 & 0.032642 & 2.476041 \\
\hline 37 & 1 & 7.917218 & 0.932685 & 3.430702 \\
\hline 38 & 6 & -7.909443 & 0.738635 & -0.081653 \\
\hline 39 & 6 & -9.078957 & 0.645949 & 0.636653 \\
\hline 40 & 6 & -9.086383 & 0.037084 & 1.913252 \\
\hline 41 & 6 & -7.918608 & -0.463598 & 2.443797 \\
\hline 42 & 1 & -7.903561 & 1.208813 & -1.067690 \\
\hline 43 & 1 & -10.007507 & 1.043380 & 0.221419 \\
\hline 44 & 1 & -10.019432 & -0.032642 & 2.476041 \\
\hline 45 & 1 & -7.917218 & -0.932685 & 3.430702 \\
\hline
\end{tabular}


Table S10. Optimised geometry calculated for $\mathbf{2}^{-}$(trans conformation) at DFT level in the gas phase (total charge $=$ -1 , spin multiplicity $=2$ ) in orthogonal Cartesian coordinate format.

\begin{tabular}{|c|c|c|c|c|}
\hline Atom number & $Z$ & $\mathrm{X}$ & $\mathrm{Y}$ & $\mathrm{Z}$ \\
\hline 1 & 6 & -4.972232 & -1.313489 & 0.648767 \\
\hline 2 & 6 & -4.525047 & -0.065771 & 0.118747 \\
\hline 3 & 6 & -5.485848 & 0.797942 & -0.390381 \\
\hline 4 & 6 & -6.865586 & 0.485796 & -0.370248 \\
\hline 5 & 6 & -7.293941 & -0.765189 & 0.175341 \\
\hline 6 & 6 & -6.301898 & -1.647739 & 0.676917 \\
\hline 7 & 6 & -3.091478 & 0.271579 & 0.121225 \\
\hline 8 & 6 & -2.634264 & 1.564223 & 0.134004 \\
\hline 9 & 16 & -0.970607 & 1.953014 & 0.137410 \\
\hline 10 & 28 & 0.000000 & 0.000000 & 0.125086 \\
\hline 11 & 16 & 0.970607 & -1.953014 & 0.137410 \\
\hline 12 & 6 & 2.634264 & -1.564223 & 0.134004 \\
\hline 13 & 6 & 3.091478 & -0.271579 & 0.121225 \\
\hline 14 & 6 & 4.525047 & 0.065771 & 0.118747 \\
\hline 15 & 6 & 5.485848 & -0.797942 & -0.390381 \\
\hline 16 & 6 & 6.865586 & -0.485796 & -0.370248 \\
\hline 17 & 6 & 7.293941 & 0.765189 & 0.175341 \\
\hline 18 & 6 & 6.301898 & 1.647739 & 0.676917 \\
\hline 19 & 6 & 4.972232 & 1.313489 & 0.648767 \\
\hline 20 & 16 & -1.922293 & -1.018473 & 0.118854 \\
\hline 21 & 16 & 1.922293 & 1.018473 & 0.118854 \\
\hline 22 & 1 & 3.331383 & -2.406037 & 0.179822 \\
\hline 23 & 1 & -3.331383 & 2.406037 & 0.179822 \\
\hline 24 & 1 & 5.177794 & -1.741596 & -0.843868 \\
\hline 25 & 1 & 6.614227 & 2.607616 & 1.095346 \\
\hline 26 & 1 & 4.221233 & 2.000216 & 1.042086 \\
\hline 27 & 1 & -5.177794 & 1.741596 & -0.843868 \\
\hline 28 & 1 & -6.614227 & -2.607616 & 1.095346 \\
\hline 29 & 1 & -4.221233 & -2.000216 & 1.042086 \\
\hline 30 & 6 & 8.676762 & 1.074530 & 0.188938 \\
\hline 31 & 6 & 9.606260 & 0.192691 & -0.315256 \\
\hline 32 & 6 & 9.185662 & -1.043261 & -0.859440 \\
\hline 33 & 6 & 7.850110 & -1.371619 & -0.886778 \\
\hline 34 & 1 & 8.994748 & 2.032070 & 0.608592 \\
\hline 35 & 1 & 10.668504 & 0.444803 & -0.297935 \\
\hline 36 & 1 & 9.926932 & -1.738178 & -1.259765 \\
\hline 37 & 1 & 7.524984 & -2.325860 & -1.307701 \\
\hline 38 & 6 & -7.850110 & 1.371619 & -0.886778 \\
\hline 39 & 6 & -9.185662 & 1.043261 & -0.859440 \\
\hline 40 & 6 & -9.606260 & -0.192691 & -0.315256 \\
\hline 41 & 6 & -8.676762 & -1.074530 & 0.188938 \\
\hline 42 & 1 & -7.524984 & 2.325860 & -1.307701 \\
\hline 43 & 1 & -9.926932 & 1.738178 & -1.259765 \\
\hline 44 & 1 & -10.668504 & -0.444803 & -0.297935 \\
\hline 45 & 1 & -8.994748 & -2.032070 & 0.608592 \\
\hline
\end{tabular}


Table S11. Selected optimized bond lengths $(\AA)$ and angles $\left(^{\circ}\right)$ for the cis and trans isomers of $\mathbf{1}^{\mathrm{x}-}(\mathrm{x}=0,1)$ at the optimized geometries in the gas phase and $\mathrm{CH} 2 \mathrm{Cl}_{2}(\mathrm{IEF}-\mathrm{PCM}$ SCRF model), compared with the corresponding structural parameters of $\mathbf{1}$ and $(\mathrm{TBA})\left(\mathbf{1}^{-}\right){ }^{\mathrm{a}}$

\begin{tabular}{|c|c|c|c|c|c|c|c|c|c|c|}
\hline & \multicolumn{8}{|c|}{ Calculated } & \multicolumn{2}{|c|}{ Experimental } \\
\hline & \multicolumn{4}{|c|}{1} & \multicolumn{4}{|c|}{$\mathbf{1}^{-}$} & $1^{b}$ & $\mathbf{1}^{-}$ \\
\hline & \multicolumn{2}{|c|}{ cis } & \multicolumn{2}{|c|}{ trans } & \multicolumn{2}{|c|}{ cis } & \multicolumn{2}{|c|}{ trans } & \multirow[b]{2}{*}{ trans } & \multirow{2}{*}{ trans } \\
\hline & Gas & $\mathrm{CH}_{2} \mathrm{Cl}_{2}$ & Gas & $\mathrm{CH}_{2} \mathrm{Cl}_{2}$ & Gas & $\mathrm{CH}_{2} \mathrm{Cl}_{2}$ & Gas & $\mathrm{CH}_{2} \mathrm{Cl}_{2}$ & & \\
\hline $\mathrm{Ni}-\mathrm{S} 1$ & 2.180 & 2.176 & 2.181 & 2.177 & 2.151 & 2.151 & 2.150 & 2.155 & 2.128 & 2.139 \\
\hline $\mathrm{Ni}-\mathrm{S} 2$ & 2.177 & 2.176 & 2.177 & 2.176 & 2.153 & 2.154 & 2.154 & 2.155 & 2.128 & 2.150 \\
\hline $\mathrm{S} 1-\mathrm{C} 1$ & 1.711 & 1.716 & 1.710 & 1.716 & 1.685 & 1.686 & 1.685 & 1.686 & 1.685 & 1.715 \\
\hline $\mathrm{S} 2-\mathrm{C} 2$ & 1.740 & 1.743 & 1.741 & 1.743 & 1.711 & 1.714 & 1.711 & 1.714 & 1.718 & 1.744 \\
\hline $\mathrm{C} 1-\mathrm{C} 2$ & 1.370 & 1.368 & 1.370 & 1.368 & 1.391 & 1.391 & 1.391 & 1.391 & 1.375 & 1.358 \\
\hline $\mathrm{S} 1-\mathrm{Ni}-\mathrm{S} 2$ & 91.45 & 91.28 & 91.46 & 91.32 & 92.10 & 91.89 & 92.10 & 91.89 & 91.65 & 91.49 \\
\hline $\mathrm{Ni}-\mathrm{S} 1-\mathrm{C} 1$ & 103.31 & 103.64 & 103.25 & 103.54 & 103.24 & 103.52 & 103.24 & 103.52 & 104.35 & 88.51 \\
\hline $\mathrm{Ni}-\mathrm{S} 2-\mathrm{C} 2$ & 104.27 & 104.55 & 104.32 & 104.52 & 104.25 & 104.43 & 104.26 & 104.45 & 104.70 & 103.98 \\
\hline $\mathrm{S} 1-\mathrm{C} 1-\mathrm{C} 2$ & 122.59 & 122.27 & 122.66 & 122.36 & 122.32 & 122.16 & 122.38 & 122.24 & 121.30 & 105.02 \\
\hline $\mathrm{S} 2-\mathrm{C} 2-\mathrm{C} 1$ & 118.38 & 118.27 & 118.30 & 118.26 & 118.09 & 118.00 & 118.01 & 117.90 & 117.80 & 117.19 \\
\hline $\mathrm{C} 1-\mathrm{C} 2-\mathrm{C} 3-\mathrm{C} 4$ & 29.90 & 28.42 & 25.84 & 28.99 & 29.88 & 28.98 & 29.60 & 28.24 & 29.00 & 18.10 \\
\hline
\end{tabular}

${ }^{\mathrm{a}}$ Numbering scheme as in Figure S9; ${ }^{\mathrm{b}}$ average of the two independent complex molecules in the unit cell. 


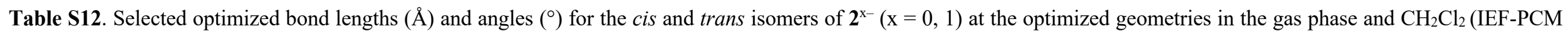
SCRF model), compared with the corresponding structural parameters of $\mathbf{2}$ and and (TBA)( $\left.2^{-}\right){ }^{a}$

\begin{tabular}{|c|c|c|c|c|c|c|c|c|c|c|}
\hline & \multicolumn{8}{|c|}{ Calculated } & \multicolumn{2}{|c|}{ Experimental $^{\mathrm{b}}$} \\
\hline & \multicolumn{4}{|c|}{2} & \multicolumn{4}{|c|}{$2^{-}$} & 2 & $\mathbf{2}^{-}$ \\
\hline & \multicolumn{2}{|c|}{ cis } & \multicolumn{2}{|c|}{ trans } & \multicolumn{2}{|c|}{ cis } & \multicolumn{2}{|c|}{ trans } & & \\
\hline & Gas & $\mathrm{CH}_{2} \mathrm{Cl}_{2}$ & Gas & $\mathrm{CH}_{2} \mathrm{Cl}_{2}$ & Gas & $\mathrm{CH}_{2} \mathrm{Cl}_{2}$ & Gas & $\mathrm{CH}_{2} \mathrm{Cl}_{2}$ & & $c l s$ \\
\hline $\mathrm{Ni}-\mathrm{S} 1$ & 2.180 & 2.176 & 2.181 & 2.177 & 2.151 & 2.152 & 2.150 & 2.150 & 2.148 & 2.123 \\
\hline $\mathrm{Ni}-\mathrm{S} 2$ & 2.177 & 2.176 & 2.175 & 2.176 & 2.153 & 2.155 & 2.154 & 2.155 & 2.146 & 2.144 \\
\hline $\mathrm{S} 1-\mathrm{C} 1$ & 1.709 & 1.715 & 1.708 & 1.715 & 1.685 & 1.685 & 1.684 & 1.685 & 1.724 & 1.681 \\
\hline $\mathrm{S} 2-\mathrm{C} 2$ & 1.740 & 1.743 & 1.741 & 1.743 & 1.712 & 1.715 & 1.713 & 1.716 & 1.726 & 1.719 \\
\hline $\mathrm{C} 1-\mathrm{C} 2$ & 1.371 & 1.369 & 1.371 & 1.369 & 1.391 & 1.391 & 1.391 & 1.391 & 1.355 & 1.381 \\
\hline $\mathrm{S} 1-\mathrm{Ni}-\mathrm{S} 2$ & 91.51 & 91.35 & 91.49 & 91.37 & 92.09 & 91.85 & 92.06 & 91.86 & 91.48 & 91.50 \\
\hline $\mathrm{Ni}-\mathrm{S} 1-\mathrm{C} 1$ & 103.32 & 103.60 & 103.27 & 103.52 & 103.26 & 103.56 & 103.28 & 103.56 & 104.25 & 104.50 \\
\hline $\mathrm{Ni}-\mathrm{S} 2-\mathrm{C} 2$ & 104.15 & 104.45 & 104.27 & 104.43 & 104.25 & 104.47 & 104.32 & 104.48 & 104.30 & 104.81 \\
\hline $\mathrm{S} 1-\mathrm{C} 1-\mathrm{C} 2$ & 122.51 & 122.26 & 122.63 & 122.31 & 122.33 & 122.23 & 122.44 & 122.29 & 119.90 & 121.90 \\
\hline $\mathrm{S} 2-\mathrm{C} 2-\mathrm{C} 1$ & 118.51 & 118.34 & 118.33 & 118.34 & 118.07 & 117.88 & 117.89 & 117.80 & 119.95 & 117.20 \\
\hline $\mathrm{C} 1-\mathrm{C} 2-\mathrm{C} 3-\mathrm{C} 4$ & 28.50 & 29.64 & 26.16 & 30.37 & 24.45 & 26.09 & 26.94 & 25.79 & 26.80 & 2.00 \\
\hline
\end{tabular}

${ }^{a}$ Numbering scheme as in Figure S10; ${ }^{\mathrm{b}}$ average between the two ligands in the complex molecule. 
Table S13. NBO charges Q (e) calculated on the central nickel atom for the cis and trans isomers of $\mathbf{1}^{\mathrm{x}-}$ and $\mathbf{2}^{\mathrm{x}-}(\mathrm{x}=0$, 1) in the gas phase and $\mathrm{CH}_{2} \mathrm{Cl}_{2}$ (IEF-PCM SCRF model).

\begin{tabular}{|c|c|c|c|}
\hline & & & $Q(\mathrm{Ni})$ \\
\hline \multirow{4}{*}{1} & \multirow[b]{2}{*}{ cis } & Gas & -0.430 \\
\hline & & $\mathrm{CH}_{2} \mathrm{Cl}_{2}$ & -0.425 \\
\hline & \multirow[b]{2}{*}{ trans } & Gas & -0.429 \\
\hline & & $\mathrm{CH}_{2} \mathrm{Cl}_{2}$ & -0.424 \\
\hline \multirow{4}{*}{$1^{-}$} & \multirow{2}{*}{ cis } & Gas & -0.367 \\
\hline & & $\mathrm{CH}_{2} \mathrm{Cl}_{2}$ & -0.419 \\
\hline & \multirow{2}{*}{ trans } & Gas & -0.367 \\
\hline & & $\mathrm{CH}_{2} \mathrm{Cl}_{2}$ & -0.417 \\
\hline \multirow{4}{*}{2} & \multirow{2}{*}{ cis } & Gas & -0.428 \\
\hline & & $\mathrm{CH}_{2} \mathrm{Cl}_{2}$ & -0.423 \\
\hline & \multirow{2}{*}{ trans } & Gas & -0.428 \\
\hline & & $\mathrm{CH}_{2} \mathrm{Cl}_{2}$ & -0.425 \\
\hline \multirow{4}{*}{$2^{-}$} & \multirow{2}{*}{ cis } & Gas & -0.366 \\
\hline & & $\mathrm{CH}_{2} \mathrm{Cl}_{2}$ & -0.417 \\
\hline & \multirow{2}{*}{ trans } & Gas & -0.366 \\
\hline & & $\mathrm{CH}_{2} \mathrm{Cl}_{2}$ & -0.418 \\
\hline
\end{tabular}


Table S14. Electronic transitions responsible for the NIR absorptions calculated for the cis and trans isomers of $\mathbf{1}^{\mathrm{x}-}$ and $\mathbf{2}^{\mathrm{x}-}(\mathrm{x}=0,1)$ in $\mathrm{CH}_{2} \mathrm{Cl}_{2}$ (IEF-PCM SCRF). For each transition, the oscillator strength $f$, excitation energy $E$ (eV), wavelength $\lambda(\mathrm{nm})$, and molecular orbital composition of the excited state functions are reported.

\begin{tabular}{lccccccc}
\hline & Isomer & Excited state & $f$ & $E$ & $\lambda$ & Composition $^{\text {a }}$ & $\%$ \\
\hline \multirow{2}{*}{$\mathbf{1}$} & cis & S & 0.430 & 1.560 & 795 & $95 \rightarrow 96$ & 94 \\
& trans & S1 & 0.502 & 1.589 & 780 & $95 \rightarrow 96$ & 96 \\
$\mathbf{1}^{-}$ & cis & D4 & 0.174 & 1.255 & 988 & $95 \rightarrow 96$ & 82 \\
& trans & D4 & 0.221 & 1.279 & 969 & $95 \rightarrow 96$ & 97 \\
$\mathbf{2}$ & cis & S1 & 0.639 & 1.438 & 862 & $121 \rightarrow 122$ & 97 \\
& trans & S1 & 0.715 & 1.460 & 849 & $121 \rightarrow 122$ & 100 \\
$\mathbf{2}^{-}$ & cis & D4 & 0.247 & 1.237 & 1002 & $121 \rightarrow 122$ & 87 \\
& trans & D4 & 0.288 & 1.257 & 986 & $121 \rightarrow 122$ & 97 \\
\hline
\end{tabular}

${ }^{\mathrm{a}}$ Molecular orbitals are labelled according to Figures 4 and S12 for $\mathbf{1}^{\mathrm{x}-}$ and $\mathbf{2}^{\mathrm{x}-}$, respectively. 
Table S15. External quantum efficiency $\eta \%$ recorded in the NIR region at different wavelengths $\lambda$ (nm) for devices based on complexes 1 and $2\left(V_{\mathrm{BIAS}}=60 \mathrm{~V}\right)$.

\begin{tabular}{ccc}
\hline$\lambda$ & $\mathbf{1}$ & $\mathbf{2}$ \\
\hline 770 & $7.00 \cdot 10^{-1}$ & $8.48 \cdot 10^{-3}$ \\
810 & $4.56 \cdot 10^{-1}$ & $8.44 \cdot 10^{-3}$ \\
840 & $3.15 \cdot 10^{-1}$ & $5.0 .10^{-3}$ \\
920 & $1.69 \cdot 10^{-1}$ & $1.11 \cdot 10^{-3}$ \\
\hline
\end{tabular}


Table S16. DC Photocurrent $\left(\mathrm{I}_{\mathrm{ph}}\right)$, dark current $\left(\mathrm{I}_{\mathrm{dark}}\right)$, and their percent ratio $(r \%)$ recorded at $770 \mathrm{~nm}$ for devices based on complexes 1 and $2\left(V_{\mathrm{BIAS}}=60 \mathrm{~V}\right)$.

\begin{tabular}{ccc}
\hline & $\mathbf{1}$ & $\mathbf{2}$ \\
\hline $\mathrm{I}_{\mathrm{ph}}$ & $4.66 \mathrm{nA}$ & $0.08 \mathrm{nA}$ \\
$\mathrm{I}_{\text {dark }}$ & $50.6 \mathrm{nA}$ & $2.50 \mathrm{nA}$ \\
$r \%$ & $9.20 \%$ & $3.20 \%$ \\
\hline
\end{tabular}




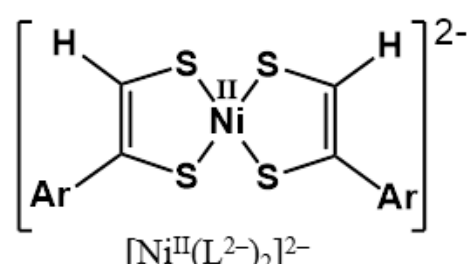

$\left[\mathrm{Ni}^{\mathrm{II}}\left(\mathrm{L}^{2-}\right.\right.$

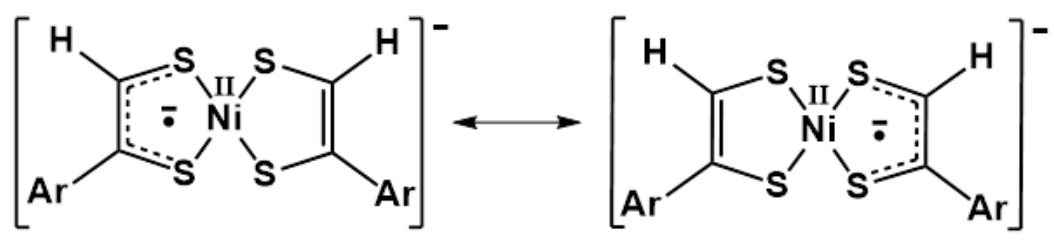

$\left[\mathrm{Ni}^{\mathrm{II}}\left(\mathrm{L}^{2-}\right)\left(\mathrm{L}^{\bullet-}\right)\right]^{-}$

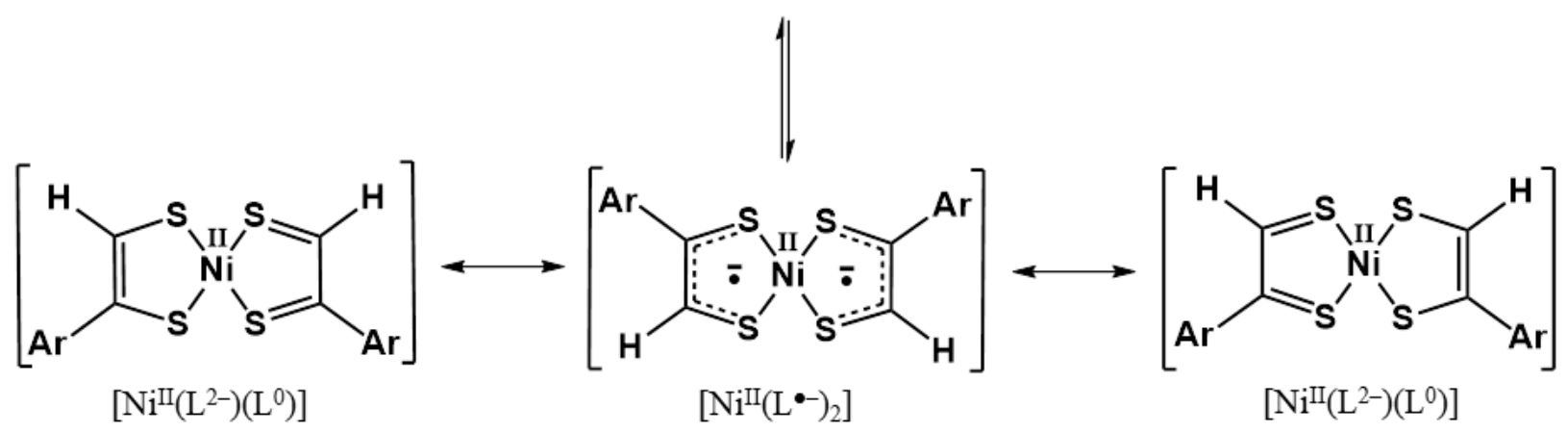

Scheme S1. Lewis structures and formal oxidation state of the central Ni atom in the redox electron transfer series of $\mathbf{1}^{\mathrm{x}-}$ and $\mathbf{2}^{\mathrm{x}-}$. 


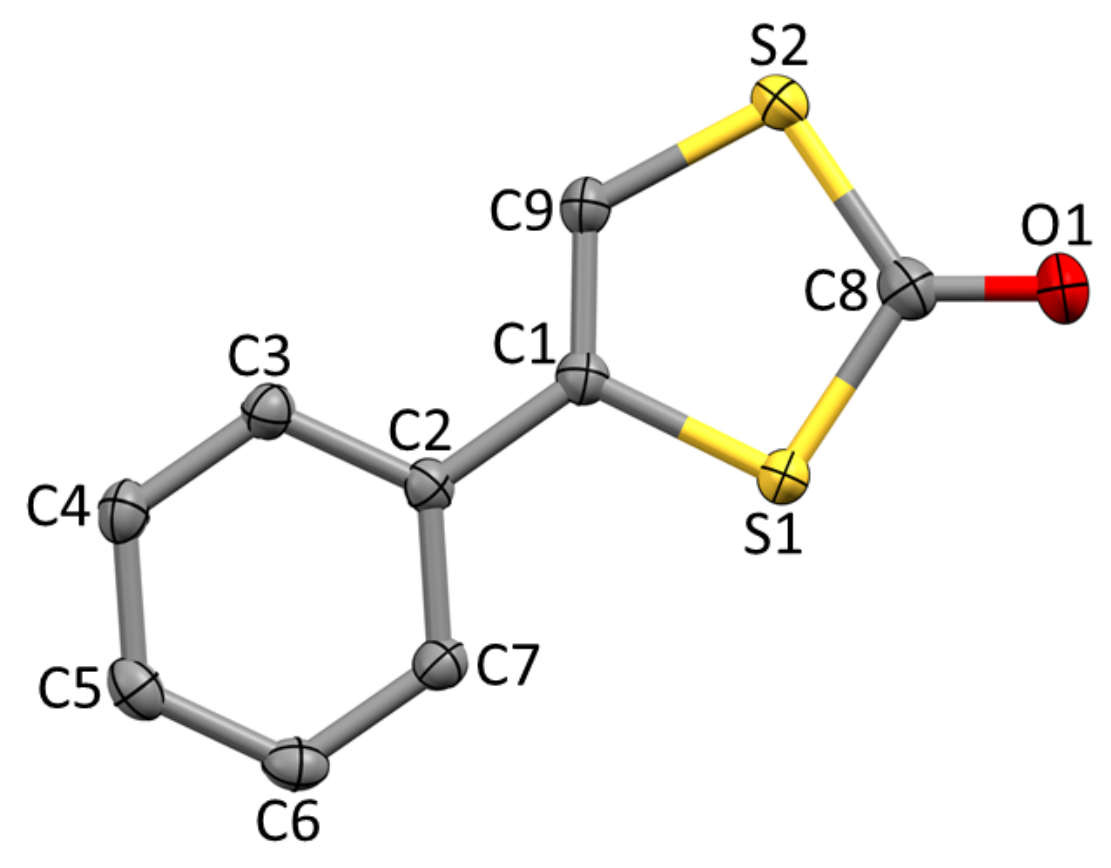

Figure S1. ORTEP drawing of 4-phenyl-[1,3]dithiol-2-one. Thermal ellipsoids are shown at the $60 \%$ probability level. Hydrogen atoms are omitted for clarity. Selected bond lengths, angles, and dihedral angles: O1-C8 1.213(3), C8-S1 1.770(2), C8-S2 1.772(2), S1-C1 1.756(2), S2-C9 1.729(2), C1-C9 1.344(3) Aं; O1-C8-S1 123.35(19), C8-S1-C1 96.98(11), S1-C1-C9 115.38(17), C1-C9-S2 119.20(17), C9-S2-C8 96.11(11) ; C9-C1-C2-C3 9.2(3) . 


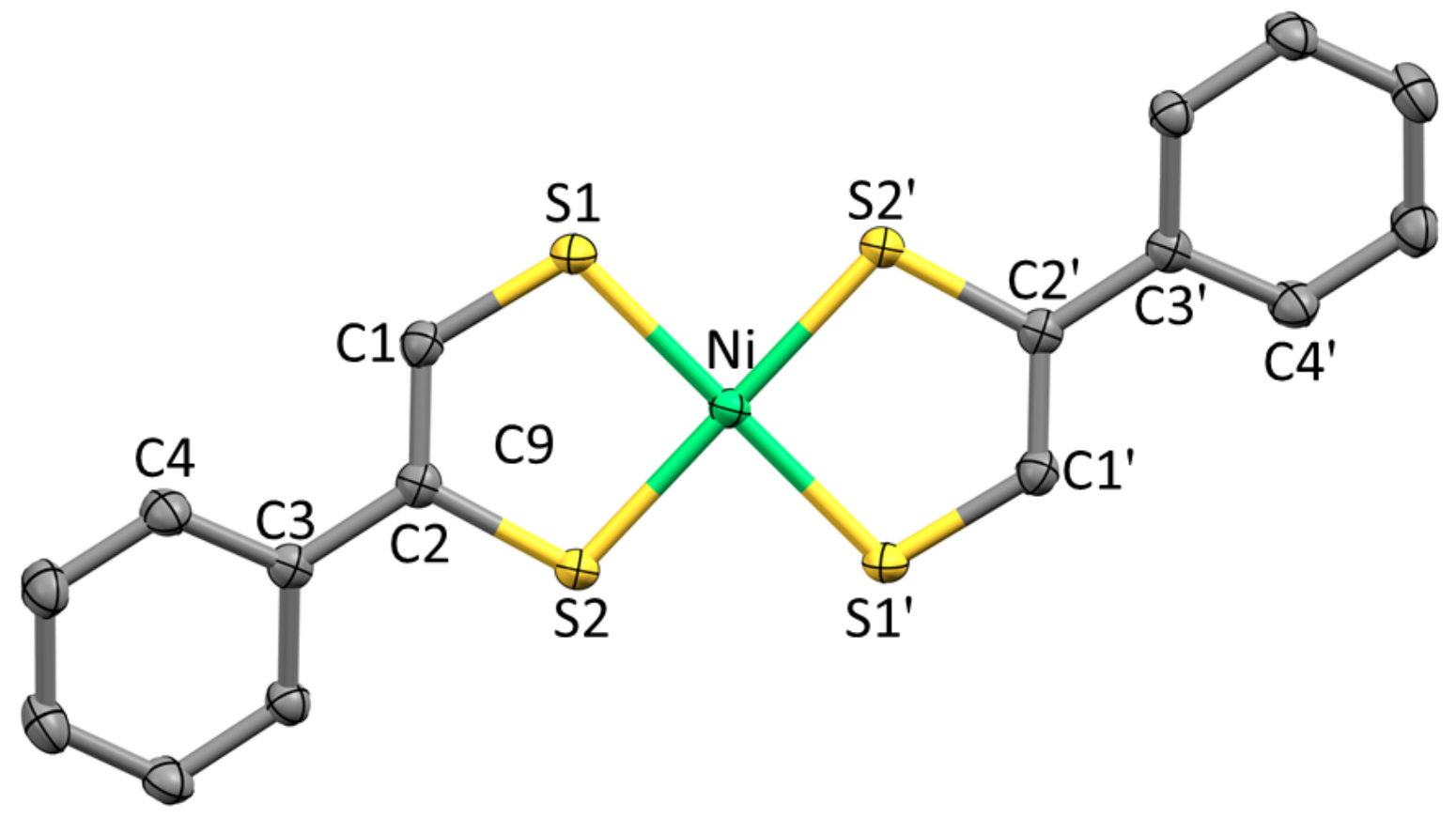

Figure S2. ORTEP drawing and atom labelling scheme for the complex anion in $\left(\mathrm{TBA}^{+}\right)\left(\mathbf{1}^{-}\right)$ viewed along the $b$ axis. Thermal ellipsoids are shown at the $60 \%$ probability level. Hydrogen atoms are omitted for clarity. Selected bond lengths, angles, and dihedral angles: Ni-S1 2.1392(4), Ni-S2 2.1495(4), S1-C1 1.7148(16), S2-C2 1.7441(16), C1-C2 1.358(2) Å; S1-Ni-S2 91.488(15), S1-Ni-S2' 88.512(15), Ni-S1-C1 103.98(6), Ni-S2-C2 105.02(5), S1-C1-C2 122.21(13), S2-C2C1 117.19(12) ${ }^{\circ}$, C1-C2-C3-C4 18.1(2), C1-S1-Ni1-S2' 177.18(6) ${ }^{\circ}$ 

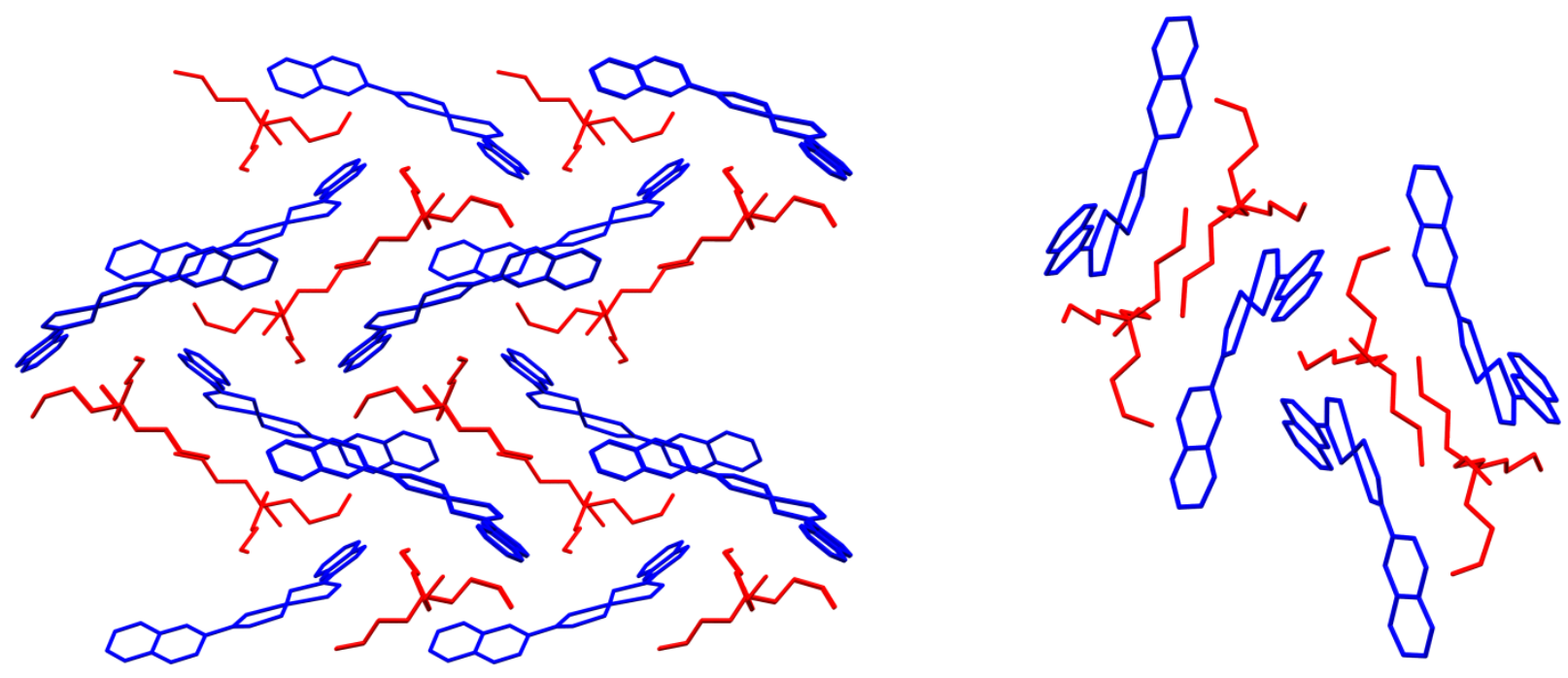

Figure S3. Drawings of portions of the crystal packing of $\left(\mathrm{TBA}^{+}\right)\left(\mathbf{2}^{-}\right)$viewed along the $a$ (left) and $b$ axis (right), showing the $\mathrm{TBA}^{+}$countercations (in red) intercalating between the $\mathbf{2}^{-}$complex molecules (in blue). Hydrogen atoms are omitted for clarity. 


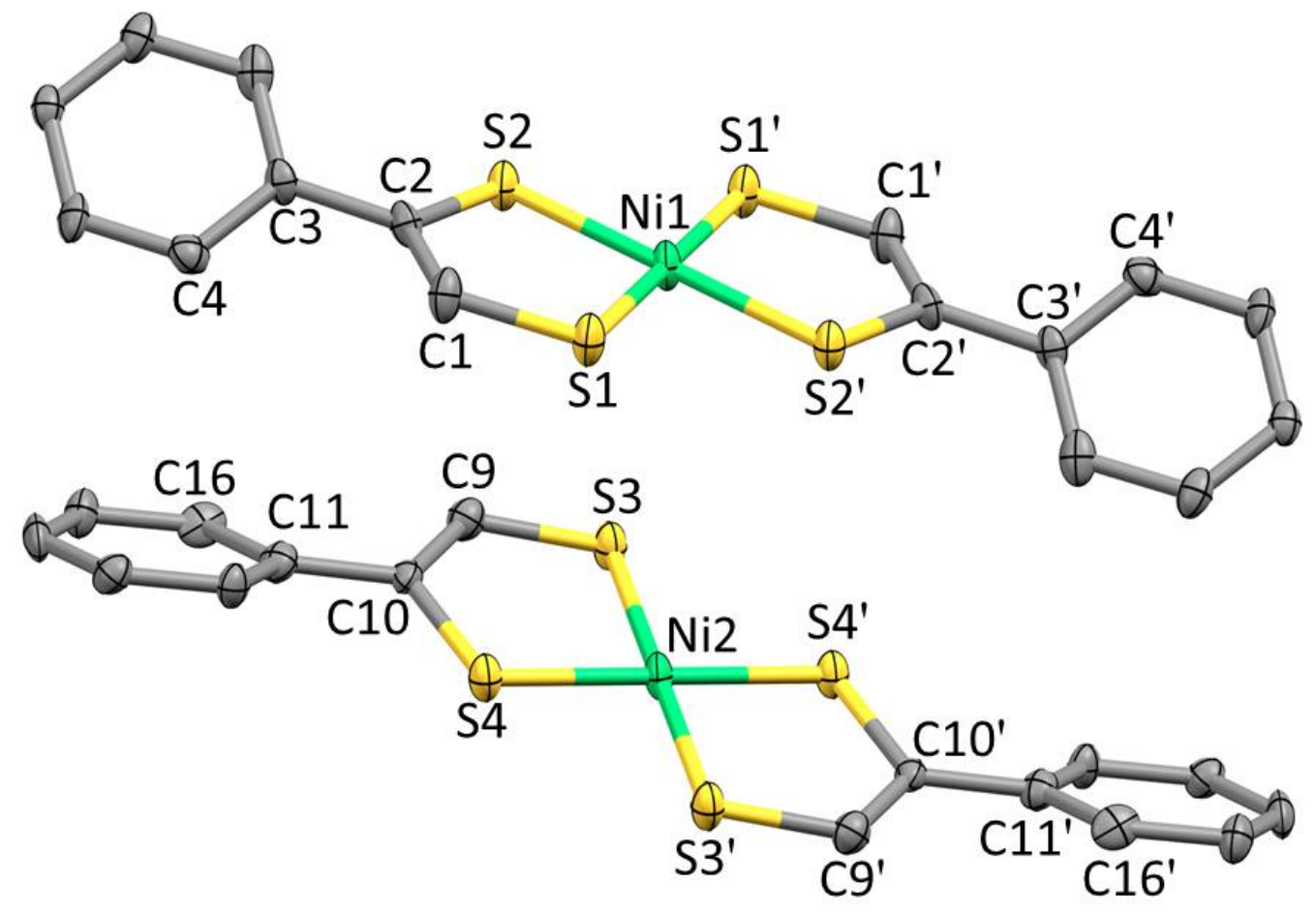

Figure S4. ORTEP drawing of the asymmetric unit of 1 viewed along the $b$ axis. Thermal ellipsoids are shown at the $60 \%$ probability level. Hydrogen atoms are omitted for clarity. Selected bond lengths, angles, and dihedral angles: Ni1-S1 2.131(2), Ni1-S2 2.129(2), S1-C1 1.692(10), S2-C2 1.711(9), C1-C2 1.376(13) Å; S1-Ni1-S2 91.67(9), S1-Ni1-S2' 88.33(9), Ni1-S1-C1 104.2(4), Ni1-S2-C2 104.9(3), S1-C1-C2 121.0(8), S2-C2-C1 118.2(8) ${ }^{\circ}$, C1-C2-C3-C4 33.6(14), C1-S1Ni1-S2' 179.5(4) ${ }^{\circ}$; Ni2-S3 2.126(2), Ni2-S4 2.128(2), S3-C9 1.679(10), S4-C10 1.726(9), C9C10 1.374(14) Å; S3-Ni-S4 91.63(9), S3-Ni2-S4' 88.37(9), Ni2-S3-C9 104.5(4), Ni2-S4-C10 104.5(4), S3-C9-C10 121.6(7), S4-C10-C9 117.4(7) ${ }^{\circ}$; C9-C10-C11-C16 24.4(15), C9-S3-Ni2S4' $179.6(4)^{\circ} .^{\prime}=1-\mathrm{x},-\mathrm{y},-\mathrm{z}$. 

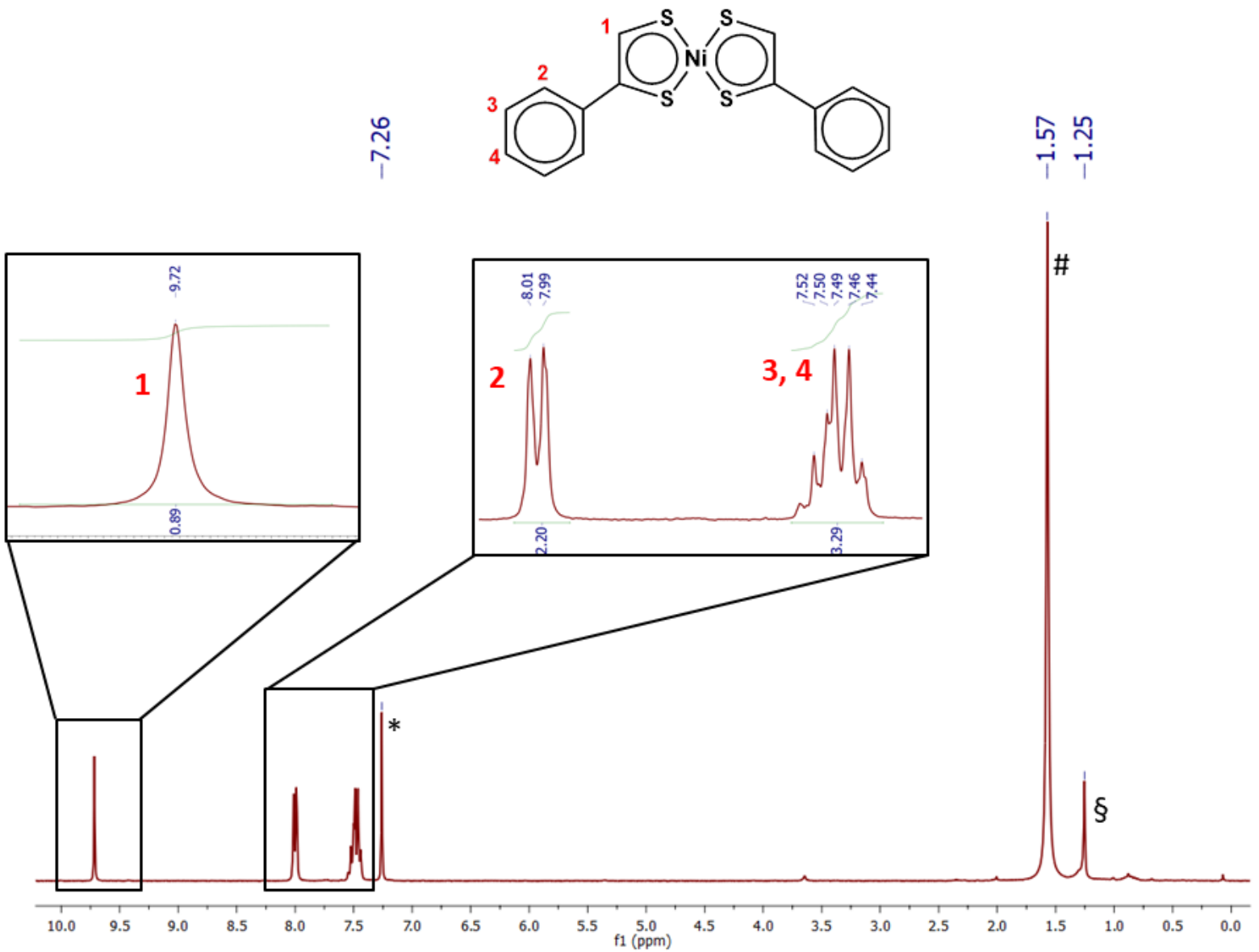

Figure S5. ${ }^{1} \mathrm{H}-\mathrm{NMR}\left(\mathrm{CDCl}_{3}, 300.13 \mathrm{MHz}\right)$ spectrum of 1. Peaks marked with an asterisk correspond to solvent $(*)$, water $(\#)$ and grease (§) signals. 

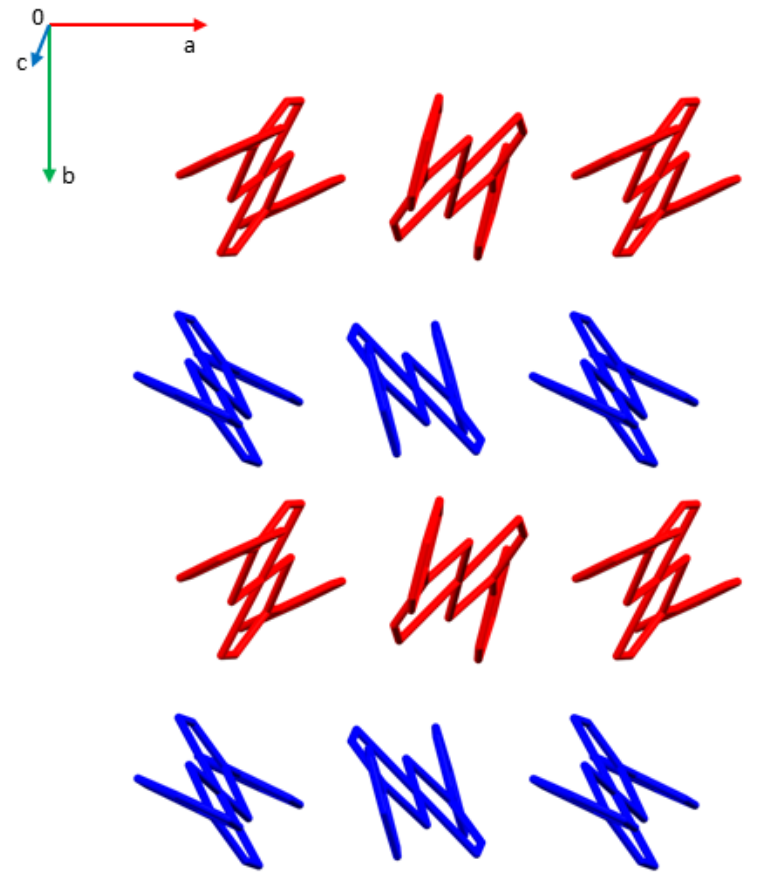

Figure S6. Drawing of a portion of the crystal packing in 1. Thermal ellipsoids are shown at the $60 \%$ probability level. Hydrogen atoms are omitted for clarity. 

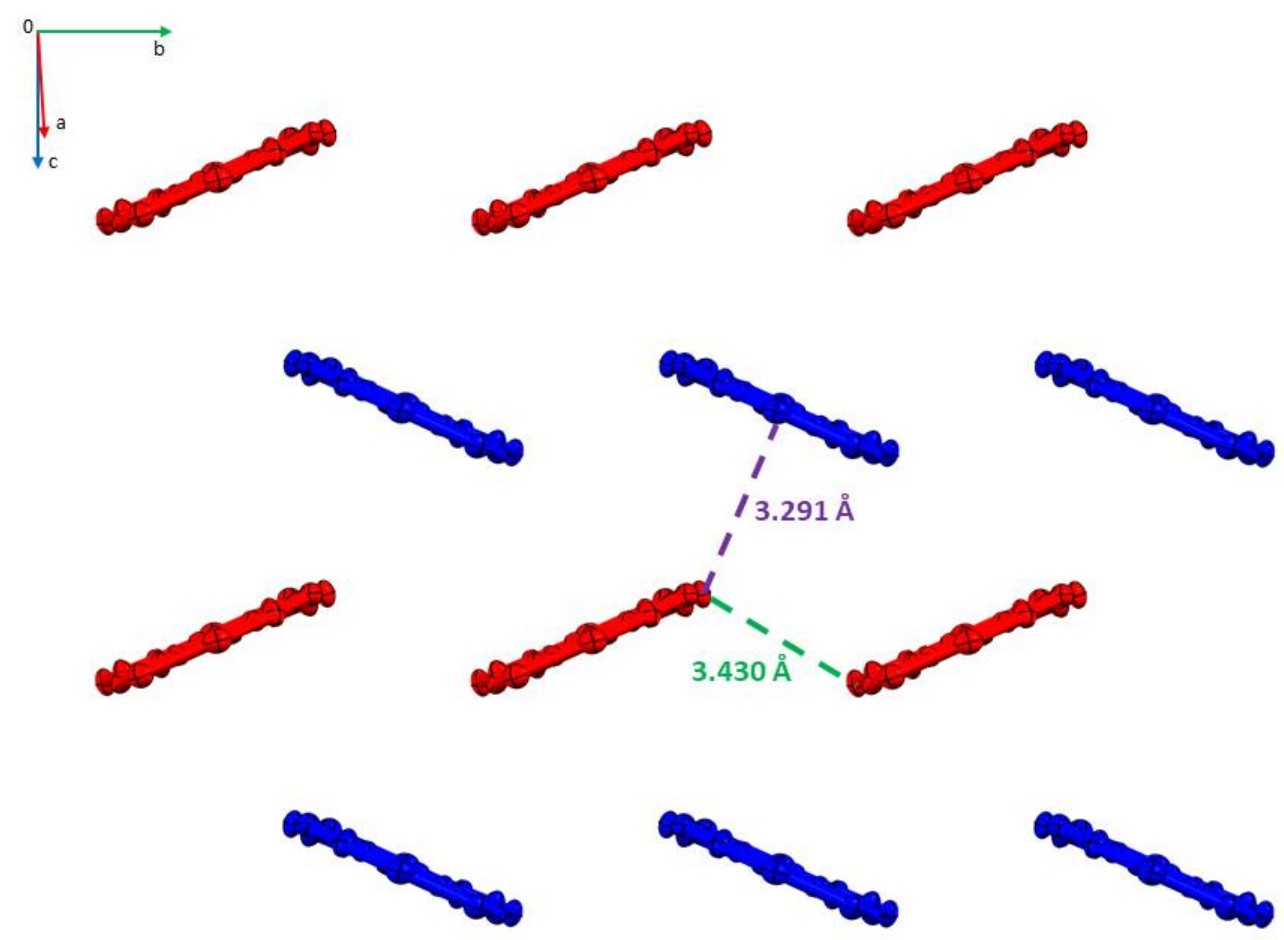

Figure S7. ORTEP drawing of a portion of the crystal packing in 2 in $\left(\mathrm{TBA}^{+}\right)\left(\mathbf{2}^{-}\right)$, showing a herringbone-type structure with alternating layers of differently oriented complex molecules in different colours (angle between the planes where the differently oriented molecules lie $=49.13^{\circ}$ ). The likewise oriented molecules belonging to one layer interact through $\mathrm{S} \cdots \mathrm{S}$ contacts (in green), while molecules belonging to different layers are held together by $\mathrm{Ni} \cdots \mathrm{S}$ contacts (in purple). Thermal ellipsoids are shown at the $60 \%$ probability level. Hydrogen atoms are omitted for clarity. 
(a)

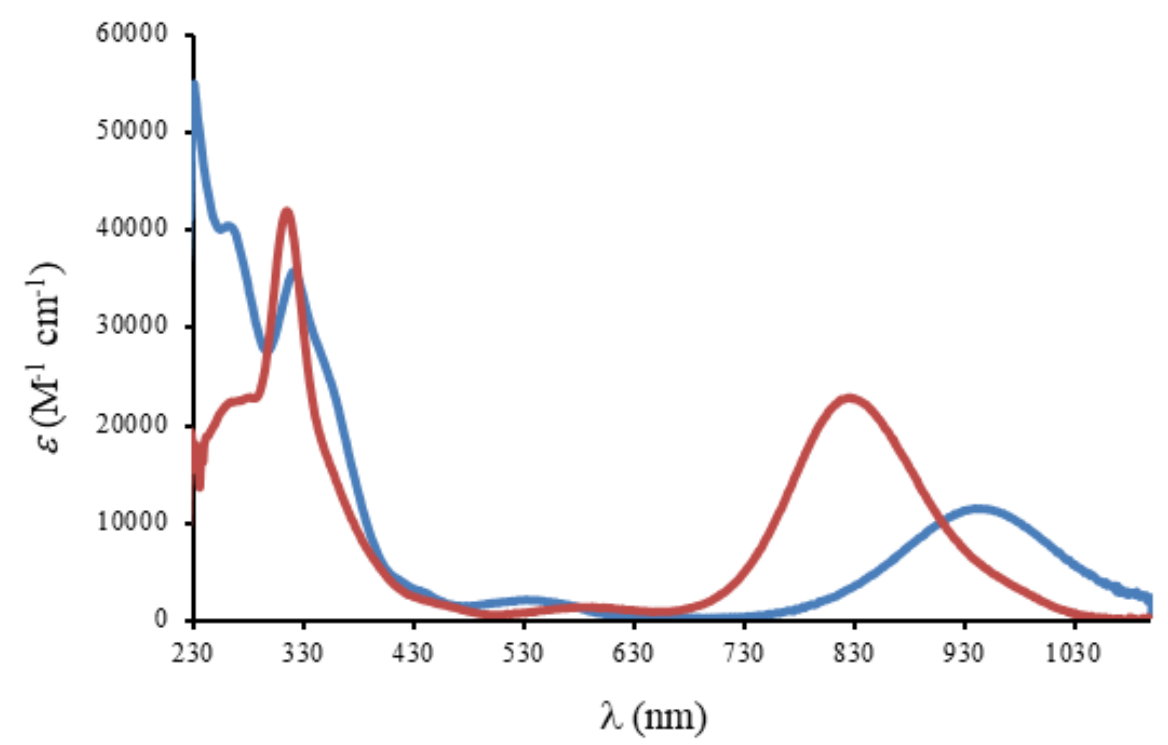

(b)

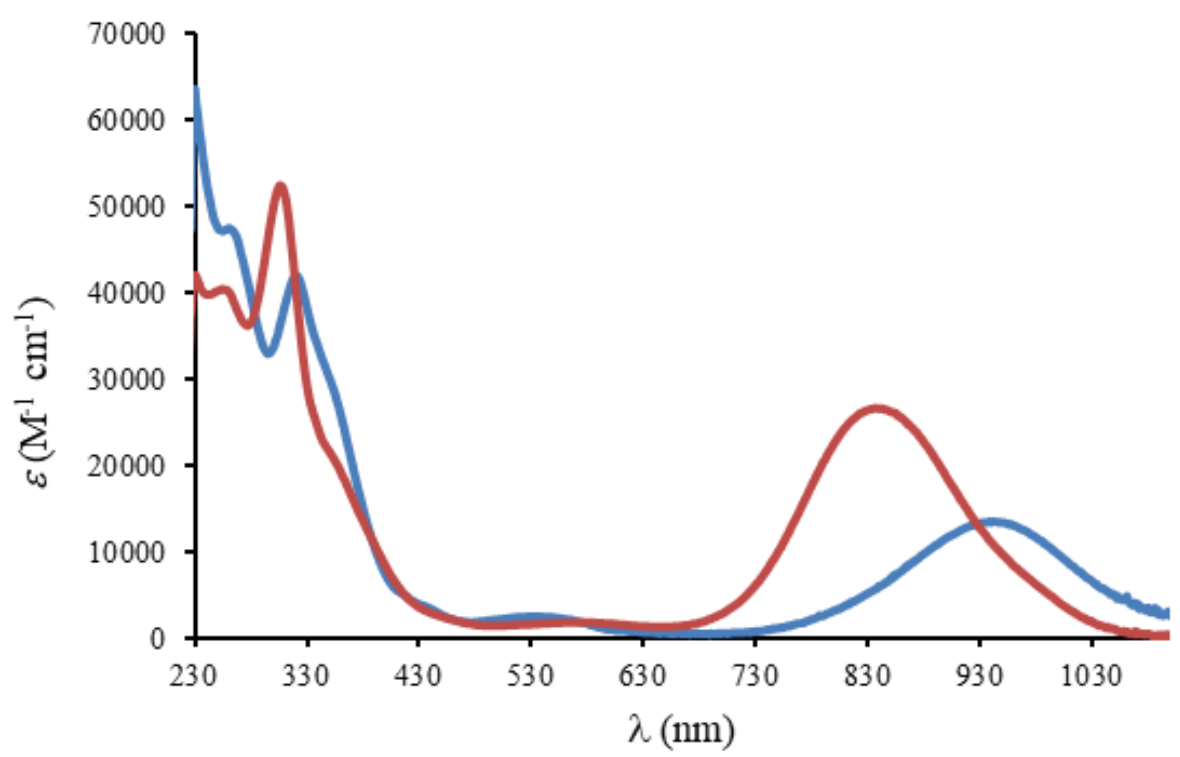

Figure S8. UV-Vis-NIR spectra of the monoanionic (blue line) and neutral forms (red line) of $\mathbf{1}^{\mathrm{x}-}$ (a) and $\mathbf{2}^{\mathrm{x}-}(\mathrm{b})$ recorded in $\mathrm{CH}_{2} \mathrm{Cl}_{2}$. 

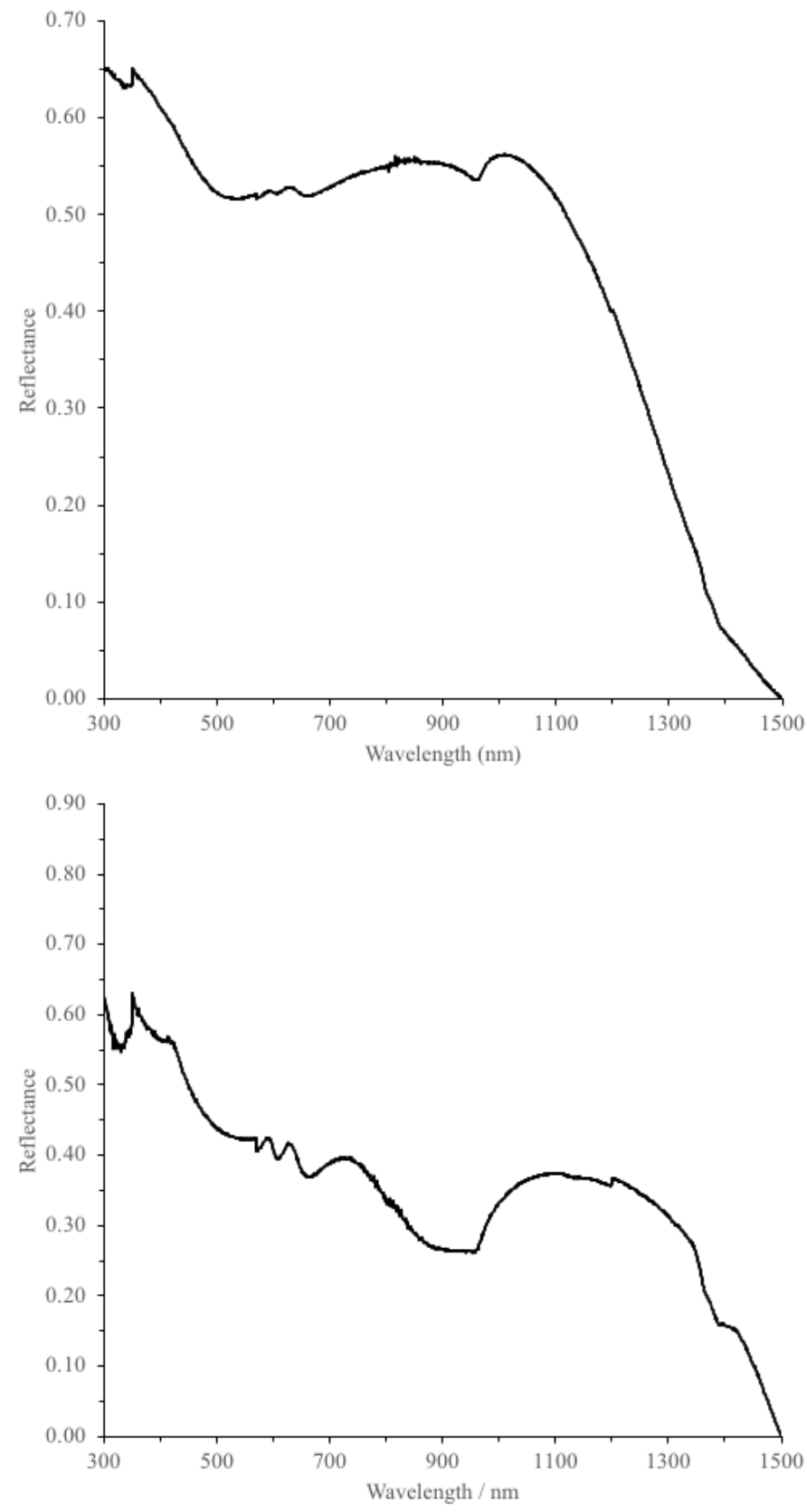

Figure S9. Solid state diffuse reflectance spectrum of 1 (top) and 2 (bottom) in the range 300-1500 nm. 

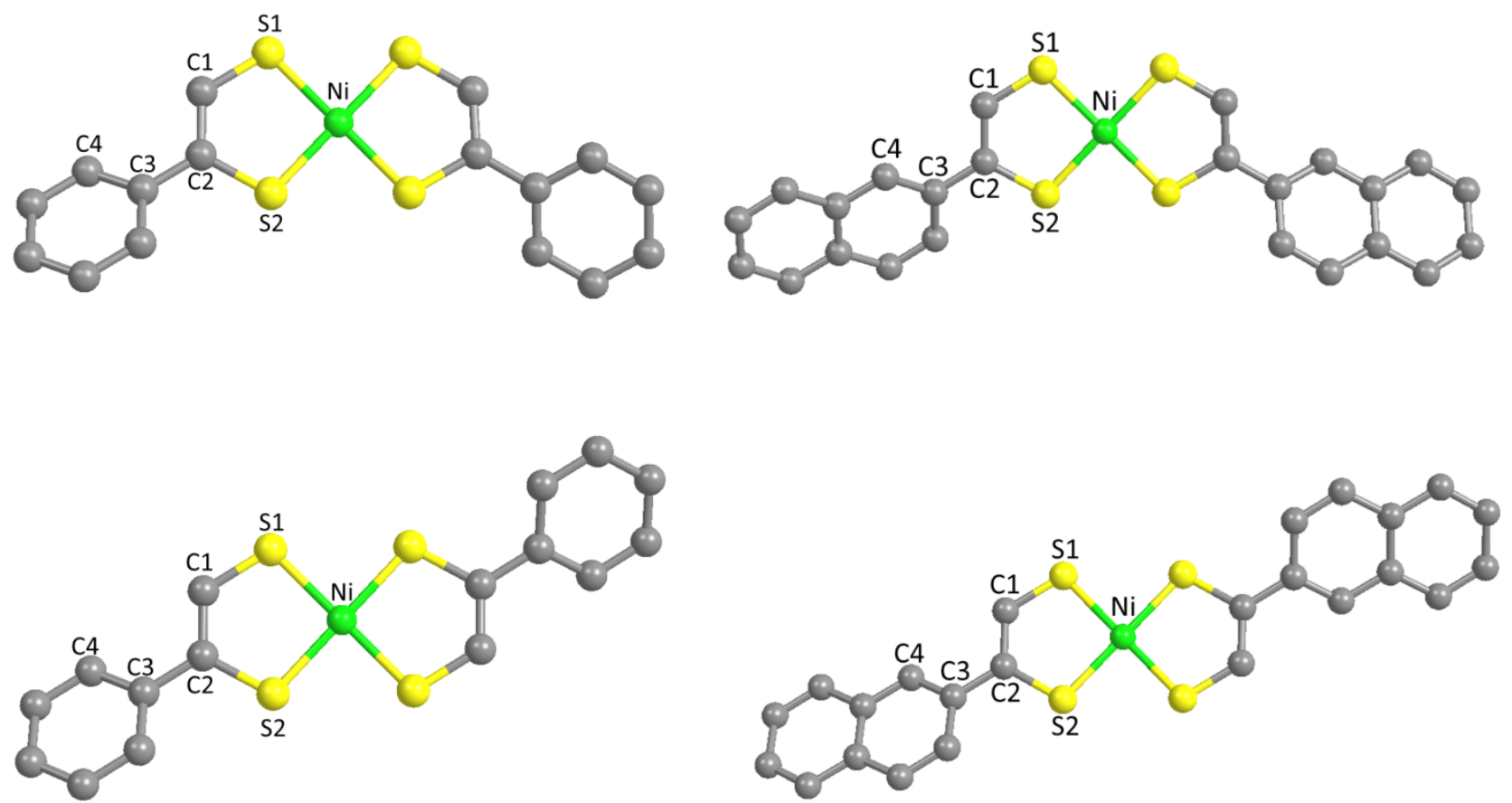

Figure S10. Molecular drawing and atom labelling scheme for 1 (left) and 2 (right) in the cis (top) and trans (bottom) conformation at the optimized geometries in the gas phase ( $C_{2}$ point group). 


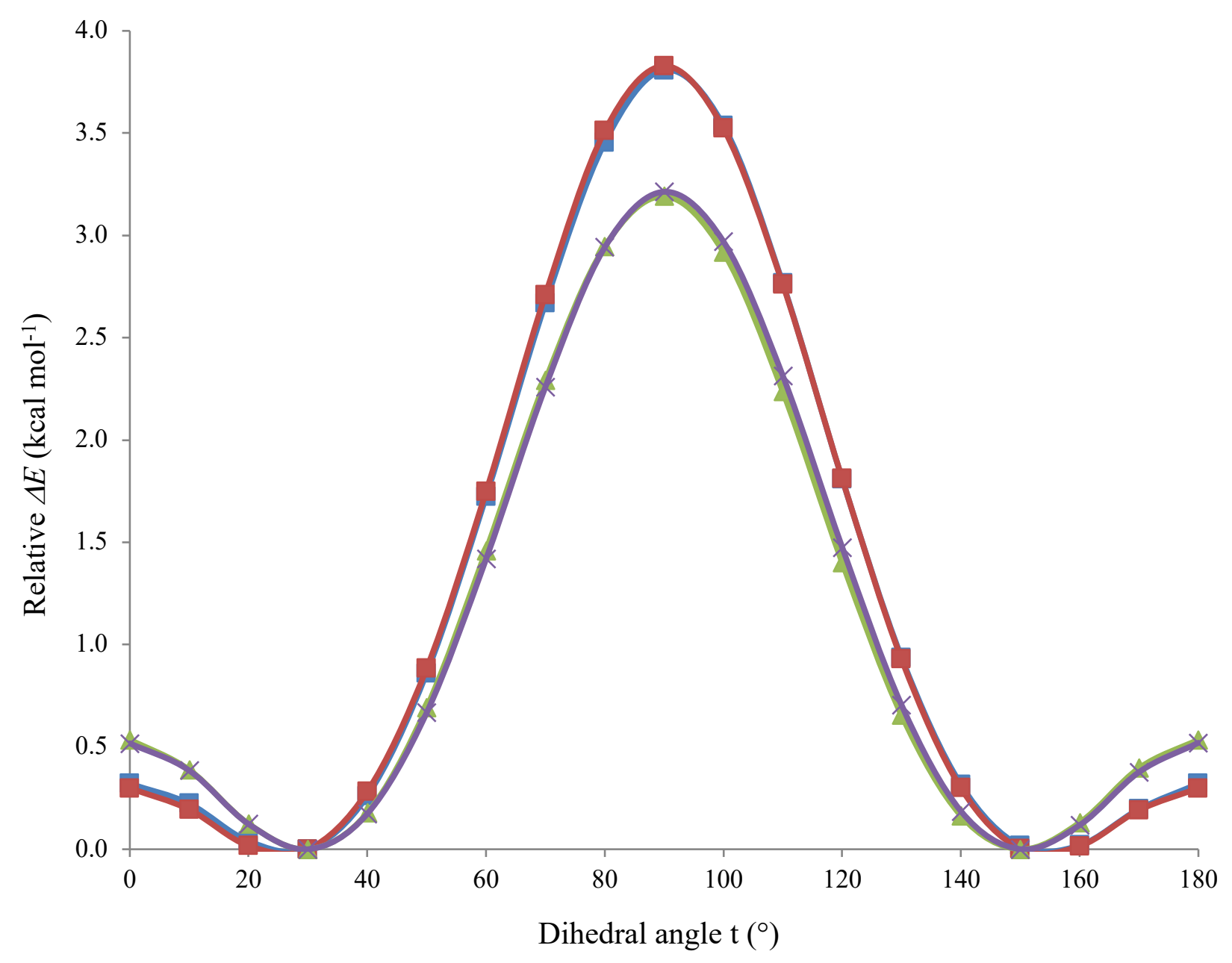

Figure S11. Relative total electronic energy variation $(\Delta E)$ calculated as a function of the phenyl rotation $(\tau)$ for the cis and trans isomers of $\mathbf{1}$ (green and purple line, respectively) and $\mathbf{1}^{-}$(blue and red line, respectively). 
$2^{-}$
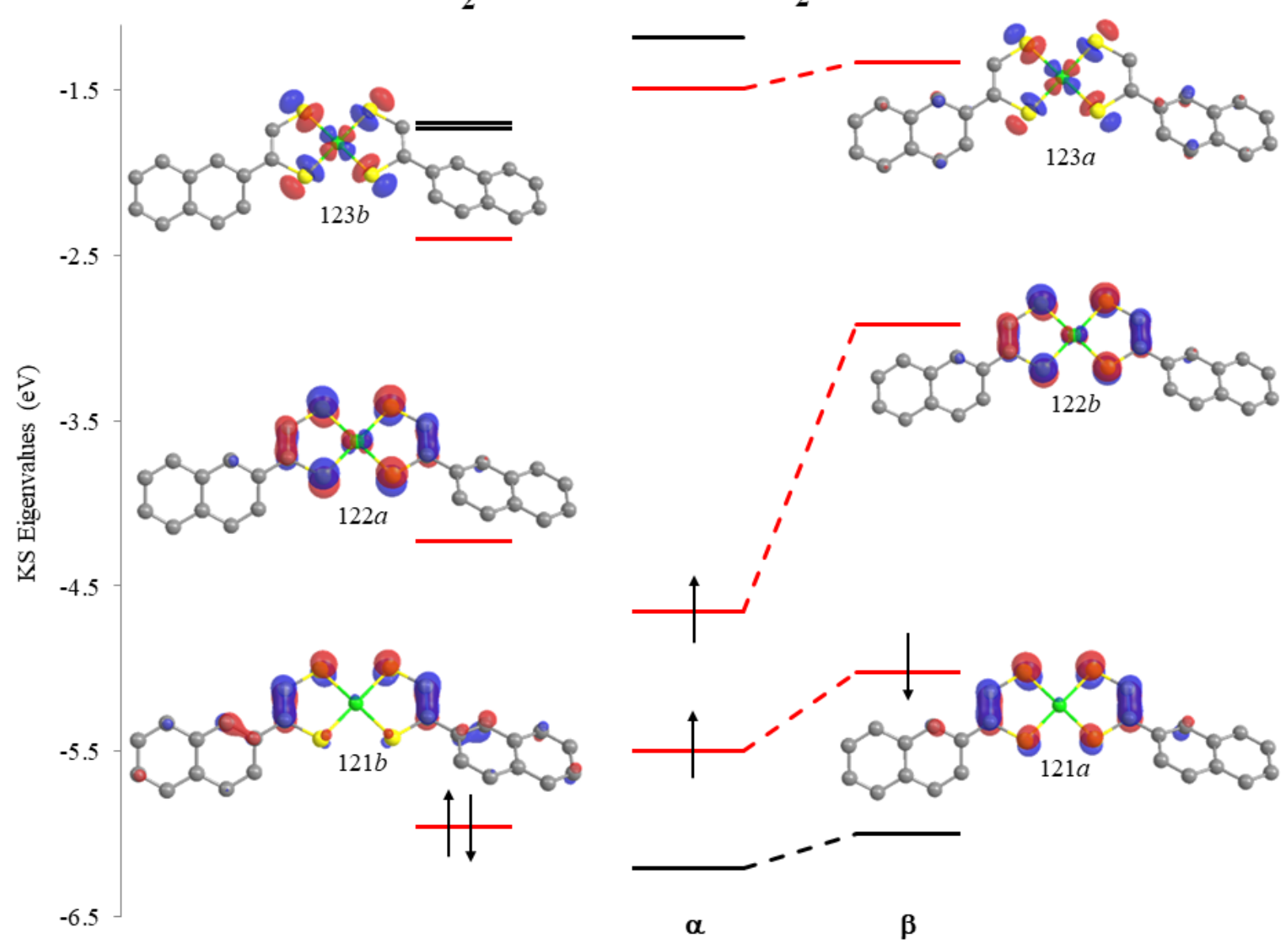

Figure S12. Correlation diagram between the $\mathrm{KS}$ eigenvalues of selected $\mathrm{MOs}(-6.5 /-1.1 \mathrm{eV})$ calculated for the cis isomers of 2 (left) and $\mathbf{2}^{-}$(right) in $\mathrm{CH}_{2} \mathrm{Cl}_{2}$ (IEF-PCM SCRF model), and drawings of the frontier KS-MOs, highlighted in red $\left(C_{2}\right.$ point group; contour value $\left.=0.05|\mathrm{e}|\right)$. 